\title{
Geometrical structures for radiation biology research as implemented in the TOPAS-nBio toolkit
}

DOI:

10.1088/1361-6560/aad8eb

Document Version

Accepted author manuscript

Link to publication record in Manchester Research Explorer

\section{Citation for published version (APA):}

Mcnamara, A. L., Ramos-méndez, J., Perl, J., Held, K., Dominguez, N., Moreno, E., Henthorn, N. T., Kirkby, K. J., Meylan, S., Villagrasa, C., Incerti, S., Faddegon, B., Paganetti, H., \& Schuemann, J. (2018). Geometrical structures for radiation biology research as implemented in the TOPAS-nBio toolkit. Physics in Medicine and Biology, 63(17), [175018]. https://doi.org/10.1088/1361-6560/aad8eb

\section{Published in:}

Physics in Medicine and Biology

\section{Citing this paper}

Please note that where the full-text provided on Manchester Research Explorer is the Author Accepted Manuscript or Proof version this may differ from the final Published version. If citing, it is advised that you check and use the publisher's definitive version.

\section{General rights}

Copyright and moral rights for the publications made accessible in the Research Explorer are retained by the authors and/or other copyright owners and it is a condition of accessing publications that users recognise and abide by the legal requirements associated with these rights.

\section{Takedown policy}

If you believe that this document breaches copyright please refer to the University of Manchester's Takedown Procedures [http://man.ac.uk/04Y6Bo] or contact uml.scholarlycommunications@manchester.ac.uk providing relevant details, so we can investigate your claim.

\section{OPEN ACCESS}




\title{
Geometrical structures for radiation biology research as implemented in the TOPAS-nBio toolkit
}

\author{
Aimee L. McNamara ${ }^{1}$, José Ramos-Méndez ${ }^{2}$, Joseph Perl ${ }^{3}$, \\ Kathryn Held ${ }^{1}$, Naoki Dominguez ${ }^{4}$, Eduardo Moreno ${ }^{4}$, \\ Nicholas T. Henthorn ${ }^{5}$, Karen J. Kirkby ${ }^{5,6}$, Sylvain Meylan ${ }^{7}$, \\ Carmen Villagrasa ${ }^{7}$, Sebastien Incerti ${ }^{8}$, Bruce Faddegon $^{2}$, \\ Harald Paganetti ${ }^{1}$ and Jan Schuemann ${ }^{1}$ \\ ${ }^{1}$ Department of Radiation Oncology, Massachusetts General Hospital, Harvard \\ Medical School, 30 Fruit St, Boston, MA 02114, USA \\ ${ }^{2}$ University of California San Francisco Comprehensive Cancer Center, San \\ Francisco, California, USA \\ ${ }^{3}$ SLAC National Accelerator Laboratory, Menlo Park, California, USA \\ ${ }^{4}$ Department of Mathematics and Physics Sciences, Benemérita Universidad \\ Autónoma de Puebla, Mexico \\ ${ }^{5}$ Division of Cancer Sciences, Faculty of Biology, Medicine and Health, University of \\ Manchester, UK \\ ${ }^{6}$ The Christie NHS Foundation Trust, Manchester, UK \\ ${ }^{7}$ IRSN, Institut de Radioprotection et de Sûreté Nucléaire, Fontenay-aux-Roses, \\ France \\ ${ }^{8}$ Université Bordeaux 1, CNRS/IN2P3, Centre d'Etudes Nucléaires de Bordeaux \\ Gradignan, CENBG, Chemin du Solarium, Gradignan, France
}

E-mail: amcnamara2@mgh.harvard.edu

\begin{abstract}
Computational simulations, such as Monte Carlo track structure simulations, offer a powerful tool for quantitatively investigating radiation interactions within cells. The modelling of the spatial distribution of energy deposition events as well as diffusion of chemical free radical species, within realistic biological geometries, can help provide a comprehensive understanding of the effects of radiation on cells. Track structure simulations, however, generally require advanced computing skills to implement. The TOPAS-nBio toolkit, an extension to TOPAS (TOol for PArticle Simulation), aims to provide users with a comprehensive framework for radiobiology simulations, without the need for advanced computing skills. This includes providing users with an extensive library of advanced, realistic, biological geometries ranging from the micrometer scale (e.g., cells and organelles) down to the nanometer scale (e.g., DNA molecules and proteins). Here we present the geometries available in TOPAS-nBio.
\end{abstract}

PACS numbers: 87.14.Gg, 87.15.Aa, 87.16.-b,87.16.Tb,87.52.-g,87.66.Jj, 87.16.Dg, 87.18.Sn

Submitted to: Phys. Med. Biol. 


\section{Introduction}

The general-purpose, open-source Geant4 Monte Carlo toolkit is frequently used for simulations in radiation therapy (Carrier et al. 2004). TOPAS (TOol for PArticle Simulation ), a wrapper to this toolkit, was designed to make Geant4 more readily available to both research and clinical medical physicists as well as to extend its functionality (Perl et al. 2012). Although TOPAS was originally designed for proton therapy applications, it has been extended to many other radiation therapy applications. TOPAS-nBio, a new TOPAS extension, further extends the functionality for radiobiology applications.

Codes such as Geant4 allow users to generate Monte Carlo simulations using a condensed history approach to predict dose on the macroscale (e.g., dose to an organ) and are typically not designed for describing particle interactions on the nanometer scale (e.g., radiation-induced DNA strand breaks). Consequently, dedicated codes (such as PARTRAC (Friedland et al. 2011), RITRACKS (Plante \& Cucinotta 2013), KURBUC (Uehara et al. 1993), etc.) or code extensions (such as Geant4-DNA (Incerti

et al. 2010, Bernal et al. 2015)) have been developed. Monte Carlo track structure simulations model step-by-step particle interactions on the nanometer scale and can be used to model the physical, physicochemical and chemical processes within biological structures. Knowledge of the spatial and temporal distribution of damage to the cell in the form of lesions, and their successive development, is needed to form a comprehensive understanding of the biological effects of radiation. Track structure simulations, however, generally require advanced computing skills to implement.

In order to address this limitation, our group has been developing a new extension to TOPAS, TOPAS-nBio, which uses and expands the Geant4-DNA toolkit. TOPASnBio has been designed as an extension to TOPAS, which is layered over the Monte Carlo simulation toolkit Geant4 (Agostinelli et al. 2003). The goal is to support radiobiology research by allowing users to simulate the effects of radiation on a subcellular level in order to interpret experimental results or to design new experiments. TOPAS-nBio has been developed to make the implementation of Monte Carlo track structure simulations for radiobiology applications easier, giving users without advanced programming skills full access to the functionality of the Geant4-DNA toolkit. TOPASnBio provides users with a catalogue of specialised biological geometries and a flexible interface to extend the reactions involved in non-homogeneous chemistry simulations (Ramos-Méndez et al. 2018). TOPAS-nBio also offers specialised scorers for biological damage (e.g., double strand breaks in DNA) and includes a set of variance reduction techniques specific to biological modelling (Ramos-Méndez et al. 2017).

An important aspect in track structure simulations is the design of realistic biological geometries in which to accurately model the spatial structure of energy deposition events from the simulated particles. Sensitive targets in radiobiological studies can cover a range of scales, from the cellular (micrometer) scale down to the molecular (nanometer) scale. In this manuscript, we describe the development and 
potential applications of the geometries in the TOPAS-nBio toolkit.

\section{Methods}

\subsection{The TOPAS toolkit}

In general, Monte Carlo simulation toolkits like Geant4 are only used by a small number of research groups with extensive coding expertise and are thus very much under-utilised in radiation oncology. In radiobiology, Monte Carlo track structure simulations are used even less due to the steep learning curve most biologists face in learning how to develop their own applications as well as the lack of present access to some of the available codes.

TOPAS is layered over Geant4 and designed to be a user code, meaning users do not need to develop code to use Geant4. TOPAS gives users access to the Geant4 toolkit libraries as well as provides additional code to extend Geant4 and make it easier to use. Users interact with TOPAS through the TOPAS parameter file system, which controls all components of the simulation including the geometry, particle source, scoring, physics, field, motion and graphical output (Perl et al. 2012). TOPAS can be downloaded from http://www.topasmc.org and documentation is provided on https://topas.readthedocs.io.

2.1.1. The TOPAS Parameter file system In order to make TOPAS reliable as well as repeatable, all simulations are built with the same compiled code. This code is written, tested and compiled by the TOPAS collaboration. Users do not write this code but use simple text parameter files to specify their simulation parameters. This file can also include the names of other parameter files to be used in the simulation. This fosters the ability to collaborate with others, since parameter files are easily shared.

The order of lines within the parameter file does not matter and other sources of error are removed by performing strict type checking and by including units for all relevant parameters. The parameter names are categorised using prefixes for the major components of the simulation, namely $G e$ for geometry components, So for particle sources, $P h$ for physics, $S c$ for scoring, $G r$ for graphics and $T s$ for overall TOPAS control. Parameter types are required using "s", "b", "i" and "d" for string, Boolean, integer or double parameters, respectively (Perl et al. 2012).

2.1.2. TOPAS extensions Although users will be able to implement most applications using the parameters files alone, some advanced users may require the added functionality of adding their own $\mathrm{C}++$ code to extend TOPAS and can do so through the TOPAS extension classes. Classes may be added for geometry components, scorers, filters, custom physics lists, particle sources or field descriptors. The extensions have been designed to be easier to implement than Geant4, with template base classes provided to the user which offer a wealth of helper functions to simplify the process. 
Users can also make use of the TOPAS parameter file system to provide additional parameters to the extension classes.

\subsection{The TOPAS-nBio extension}

TOPAS-nBio extends TOPAS to allow users to easily design Monte Carlo simulations for radiobiology applications. Following the same design philosophy as TOPAS, TOPASnBio users do not need to write code or have a detailed understanding of Geant4 or Geant4-DNA. TOPAS-nBio is an extension to TOPAS and users require the full TOPAS toolkit to run the TOPAS-nBio simulations. The geometries included in TOPAS-nBio are open-source extension classes which are fully controlled by the parameter file system. However, advanced users have the freedom to edit the currently available geometries in the extension classes or to design their own geometries and/or scorers.

\subsubsection{TOPAS-nBio Parameter file system TOPAS-nBio adopts the same easy-to-use} parameter file system as TOPAS. Figure 1 shows an example parameter file for specifying a TOPAS-nBio simulation of a fibroblast, containing a nucleus and mitochondria. The cell geometry is specified by the type "TsFibroblast1", the name of the TOPAS-nBio geometry extension class. The user can include organelles, in this case a nucleus and 10 mitochondria are placed within the cell. Users have the option of specifying some of the parameters of the cell, such as the organelle size and position within the cell.

\subsubsection{The TOPAS-nBio geometry library To cover a wide range of geometries,} TOPAS-nBio offers the user a vast catalogue of biological targets. On the micrometer scale, users can select cells by shape or differentiation, choosing to model a single cell or multiple cells (e.g., neuron network or cells in vitro). Other micrometer (sub-cellular) scale targets, such as the cell nucleus or mitochondria are also offered as geometries. A single organelle structure may be simulated or multiple organelles can be included in the full cell geometry.

On the nanometer scale, biologically significant molecules (e.g., DNA, RNA, membrane lipids) are offered as geometries. Since DNA is the primary target of radiation in the cell, realistic DNA models are important for track structure simulations. For this reason, TOPAS-nBio has developed numerous models of DNA in different stages of hierarchal folding within the cell. This includes models of the cell nucleus incorporating the DNA structure from chromatin territories and chromatin fibre models down to the double strand DNA helix. Other potential radiation targets are also considered, for example mitochondrial DNA, membranes and other molecules essential to healthy cell function (RNA, proteins).

Each geometry has its own extension class. Users may interact exclusively with the class through the parameter system or may edit the geometry within the class itself. The name of the geometry is the same as the extension class name, and users will evoke the geometry in the parameter file system using "Type" e.g. s:Ge/MyCell/Type= 

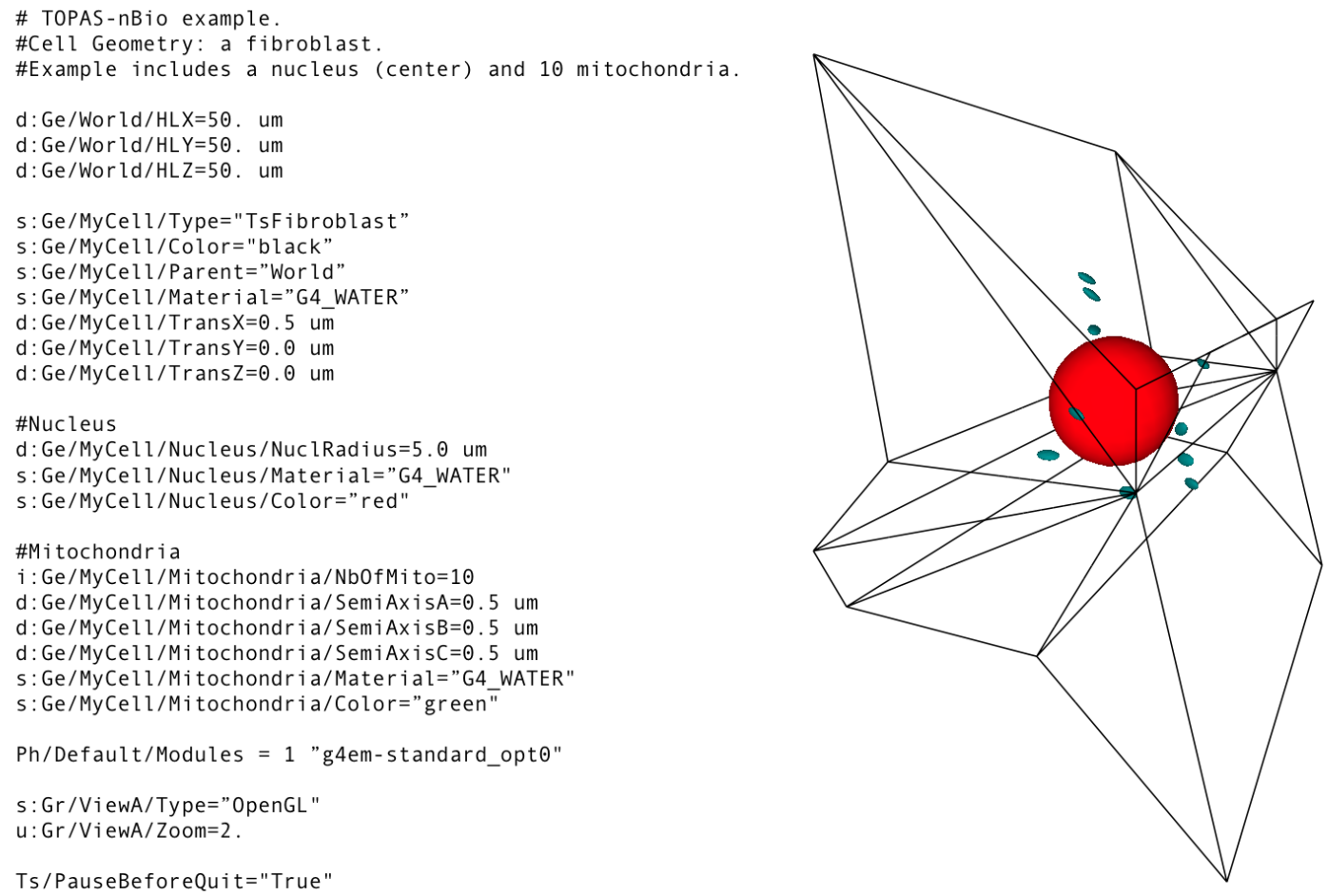

Figure 1. An example of the TOPAS parameter file system for a TOPAS-nBio geometry of a fibroblast containing a spherical nucleus (red) and ellipsoid mitochondria (green). Users may specify the position of the cell in their world volume as well as the material (in this case water) and visualisation parameters (e.g., colour). Parameters for the nucleus and mitochondria are also specified in the parameter file. The nucleus radius, organelle materials and the semi-axis lengths of the mitochondria ellipsoids are specified.

"TsFibroblast1" (see Figure 1). Each geometry type may also include additional parameters that can be changed or specified within the parameter file system e.g., number and placement of organelles or number of base-pairs (bps) in a DNA strand.

2.2.3. Materials and Physics While cells and their components are made up of materials different from pure water, the physical processes for elastic and inelastic interactions included in Geant4-DNA, and generally in most of the Monte Carlo track structure codes, were analytically developed or obtained from scaled experimental measurements in water vapour to be used in condensed-phase water medium (Incerti et al. 2010). Thus, the detailed geometrical models provided in the TOPAS-nBio suite should be composed of water (specifically "G4_WATER") when using the Geant4DNA physical processes. However, Geant4-DNA and TOPAS-nBio both offer users the possibility of creating simulations using the physical processes of condensed-history 
transport combined with the track-structure transport models, in specific regions of interest. For example, this allows one to model a gold nanoparticle (GNP) in a cell by using the low energy electromagnetic physics processes for gold inside the GNP (either based on the Penelope or Livermore libraries) and the Geant4-DNA track structure simulations in the cell regions. Condensed-history transport has the advantage of being faster and allows the inclusion of different materials, while the track-structure transport only recognises water material with the option of a re-scaled water density. Users do have the option of modelling cells and organelles with condensed history simulations using their own defined materials for the components of the cell (McNamara et al. 2016). In addition, new developments in Geant4-DNA include electron and proton inelastic (and elastic for electrons) cross sections for DNA materials namely tetrahydrofuran, trimethylphosphate, pyrimidine and purine (Bug et al. 2017), these are available through physics lists configurable with the parameter system of TOPAS. As new material crosssections for low energies become available in Geant4-DNA, these will be available for use in the TOPAS-nBio toolkit. Most other track structure Monte Carlo simulations codes do not model geometries but simply overlay structures after simulating tracks within a liquid water box. In contrast, TOPAS-nBio scores within the geometry sub-components themselves and thus allows the possibility to assign different material properties (e.g. chemical composition and density) to each structure.

The Geant4-DNA physics processes are valid for electrons, protons, some ions $(\mathrm{H}$, $\mathrm{He}, \mathrm{Li}, \mathrm{Be}, \mathrm{B}, \mathrm{C}, \mathrm{N}, \mathrm{O}, \mathrm{Si}, \mathrm{Fe}$ ) and gammas. Gamma interactions are based on the Geant4 Livermore/EADL97 models. The energy ranges valid for the particles and type of model used depends on the modular physics list used in the simulation. TOPASnBio provides users with the default G4EmDNAPhysics Geant4-DNA physics module as well as the G4EmDNAPhysics_optionX modules, where $\mathrm{X}$ can be 1 to 6 . These modules use a different combination of the elastic and inelastic models available in Geant4-DNA (Incerti et al. 2018). Advanced users have the option of writing their own physics modules and using this in TOPAS through the extensions interface. Most of the Geant4-DNA models are valid for particle interactions down to $\sim 10 \mathrm{eV}$ or less. Users may also choose to simulate particles with the low energy electromagnetic physics processes using either the Livermore physics models or the Penelope physics models which are valid for electron and photon interactions down to $\sim 250 \mathrm{eV}$.

\section{Results and Discussion}

Here we present the geometry classes of the TOPAS-nBio toolkit. The geometries have been sorted from largest (cells, micrometer scale) to smallest (molecules, nanometer scale). We describe the design of each geometry and also discuss possible applications for each.

3.0.1. Cells A common end point of measurement for cells cultured in vitro in radiobiology is the reproductive death of a cell. A cell survival curve describes 
the relationship between the radiation dose and the proportion of cells that survive. Survival, however, does not necessarily refer to cell death (apoptosis) but can refer to a multitude of other conditions. For non-proliferating cells (e.g., differentiated cells such as neurons), survival can be defined as the loss of a specific function (e.g., ability to form a working synaptic connection), while for proliferating cells (e.g., intestinal epithelium) survival may be defined as the loss of the ability to divide and produce new cells.

Monte Carlo simulations in a single cell model can provide insight into the distribution of dose in the entire cell or a specific sub-region of the cell (e.g., the cell nucleus). This may be correlated with experimental measurements of the loss of a specific function or cell apoptosis. Single cell models can also form the geometric boundary for more complex studies, for example, calculating the energy deposited in other sub-cellular components (e.g., organelles, cell membranes) or investigating DNA damage in the nucleus. TOPAS-nBio provides users with a unique framework for designing multiple cell types and the option of including organelle sub-components. Below we outline some of the cells available in TOPAS-nBio by shape.

3.0.2. Spherical, elliptical and cuboidal cells Spherical or elliptical cells are commonly used in Monte Carlo simulations (Byrne et al. 2015, Douglass et al. 2012, Incerti et al. 2016, for example). Although spherical cells seem simplistic, there are many cases where spheroid cells effectively represent realistic cells, for example a lymphocyte in suspension (Rosenbluth et al. 2006), some hepatoma cell lines (e.g., spherical FLC-4 cells (Laurent et al. 2012)) or yeast cells (Jorgensen et al. 2007). Cuboidal or columnar cells are found in kidney tubules, glandular ducts, ovaries, and the thyroid gland, usually with a central spherical nucleus, while most bacteria or blood cells are elliptical in shape. The morphology of cells can differ significantly between the in vivo and in vitro case and the user should decide on which shape accurately represents the case they wish to simulate.

Spherical, ellipsoid and cuboidal/columnar cells are modelled using the TsSphericalCell, TsEllipsoidCell and TsCuboidalCell extensions. In each case, the user can specify the size of the cell and has the option of including organelles within the cell (nucleus and/or mitochondria). Figure 2 shows examples of (a) spherical, (b) ellipsoid, (c) cuboidal and (d) columnar cell geometries, all containing a nucleus and mitochondria. Also shown is an example of spherical cells randomly distributed within a volume (e) using the TsCellCulture class. In this class, users can randomly distribute cells within a volume, representing cells in culture for example. Additional simple cell shapes are offered in the toolkit such as cylindrical and trapezoid cells (TsCylindricalCell, TsTrapezoidCell) but are not shown here.

3.0.3. Irregular cells - fibroblasts Fibroblasts are large, irregularly shaped cells and one of the most common connective tissue cell in mammals. Fibroblasts are also commonly used in radiobiology studies, from both healthy and diseased donors. Additionally, carcinoma-associated fibroblasts (CAFs) are present in most solid tumours and may 
(a)

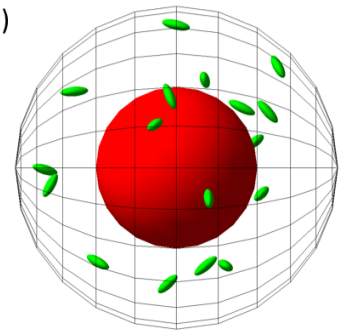

(c)

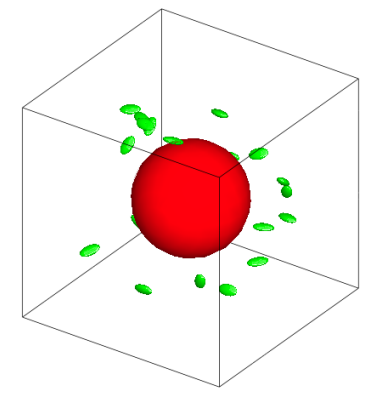

(b)

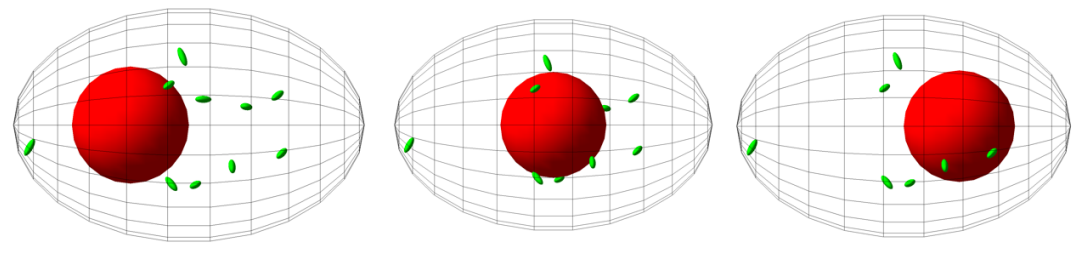

(d)

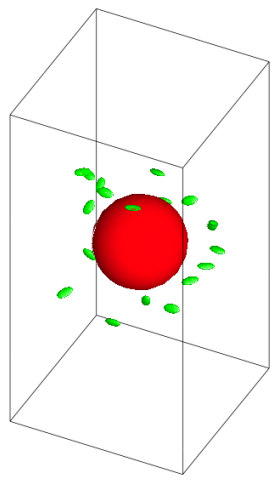

(e)

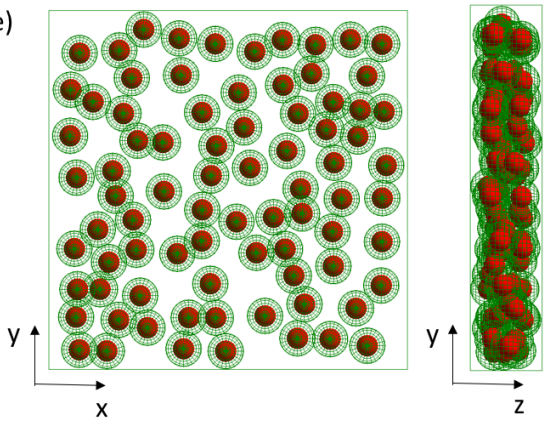

Figure 2. TOPAS-nBio cell geometries: (a) Spherical cell geometry containing a nucleus (red) and mitochondria (green), (b) ellipsoid cell geometry illustrating three different placements of the nucleus, (c) a cuboidal cell geometry, (d) columnar cell geometry and (e) spherical cells randomly distributed within a rectangular volume, representing cells in culture.

affect tumourigenesis and therapeutic response; thus understanding the CAF radiation response may be important to improve radiotherapy outcomes (Grinde et al. 2017). CAFs or myofibroblasts within the tumour may not only promote tumour growth but may also affect metastasis (Liao et al. 2009).

In TOPAS-nBio, users are given the option of using three fibroblast geometries; TsFibroblast1, TsFibroblast2 or TsFibroblast3 (see Figure 3). Users may also specify their own spatial coordinates of the points creating the cell to form a unique cell shape in the parameter file. The option of including organelles within the cell model is also available as shown in Figure 3.

3.0.4. Specialised cells - Neurons Irradiation of the central nervous system (CNS) induces varying degrees of damage to the normal tissue which may manifest in debilitating cognitive dysfunction (Gorlia et al. 2012, Saury \& Emanuelson 2011, Meyers 2000, Greene-Schloesser et al. 2012). Furthering our understanding of the underlying 

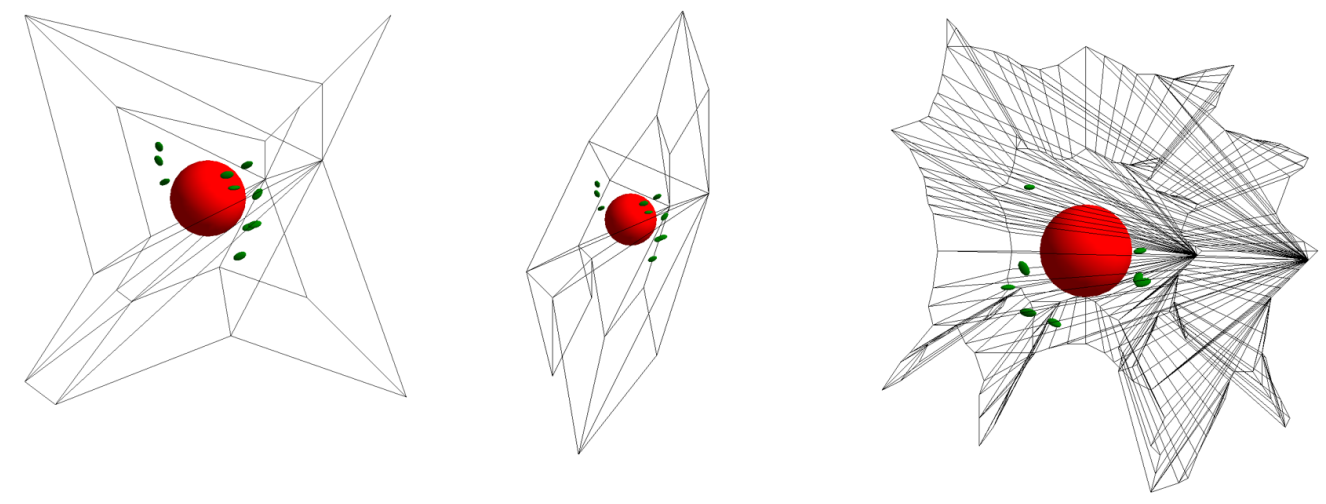

Figure 3. An example of three fibroblast geometries. Each cell model contains organelles, a nucleus (red) and mitochondria (green).

mechanisms of ionising radiation-induced damage in neurons and other cells in the CNS is thus essential for improving brain tumour patient outcomes (Makale et al. 2017) and for protecting astronauts on missions outside the orbit of earth (Parihar et al. 2015a).

The CNS is primarily made up of neurons, which are highly differentiated cells with complex tree-like structures. Generally, a neuron consists of three different regions: a cell body or soma, dendrites and an axon. Axons are specialised for the conduction of action potentials (electric impulses) from the soma while dendrites are specialised to receive chemical signals. The site where neurons communicate with each other or other cells is known as a synapse, with chemical synapses involving the transfer of neurotransmitters being the most common. The soma contains the nucleus and is the site of the synthesis of the majority of neuronal proteins. Proteins and membranes required for the renewal of the neuron are synthesised in the soma and then transported to different regions in the cell (e.g., axon). Some proteins are synthesised in dendrites via ribosomes but proteins are not generated in axons. Dendrites additionally contain organelles (e.g., dendritic mitochondria) as well as spines, small protrusions on the dendritic membrane which aid in storing and transmitting neurotransmitters. In situ neurons co-exist with supporting glial cells such as microglia, oligodendrocytes and astrocytes, which maintain homeostasis and communicate with neurons. Possible radiation targets in the neuron include the site of the genome i.e. the nucleus in the soma (Alp et al. 2015, Batmunkh et al. 2015). Other possible targets include organelles (e.g., mitochondria), structures such as spines or the cell membranes and ion channels in the dendrites (Puspitasari et al. 2016). Support glial cells such as astrocytes could also be a radiation target (Belka et al. 2001).

In order to model realistic representations of the numerous types of neurons and 


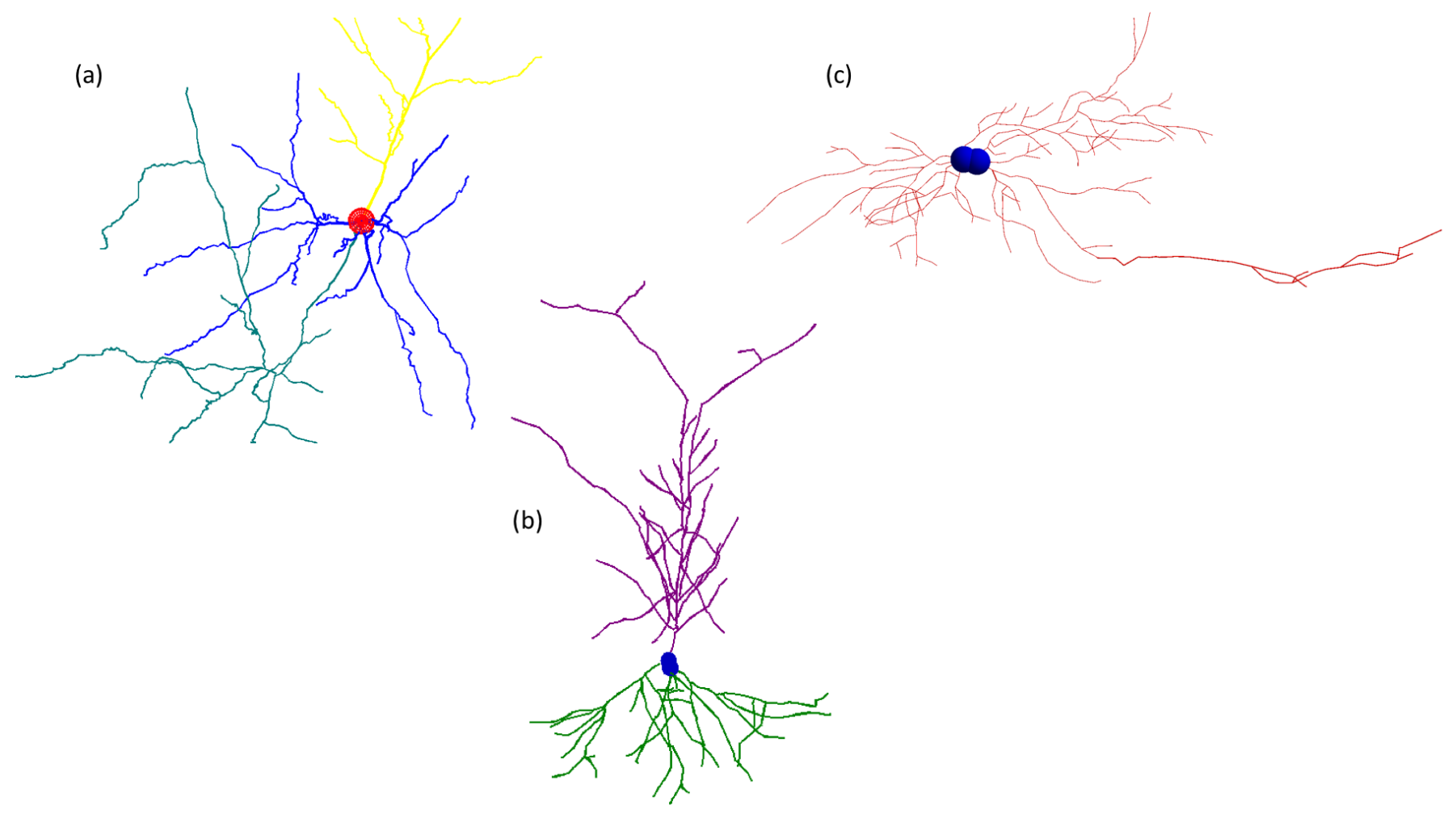

Figure 4. Three neuron morphologies from the NeuroMorpho database in TOPASnBio: (a) mouse pyramidal neuron from the occipital neocortex with a spherical soma (red), axon (yellow) and dendrites (green and blue) (b) pyramidal neuron from a mouse hippocampus with a soma consisting of two points (blue) and dendrites (green and purple) without an axon, and (c) mouse basal ganglia with a soma (blue) and dendrites (red).

supporting glial cells in the CNS, we developed an interface to the NeuroMorpho neuron database (www . neuromorpho.org) for TOPAS-nBio. The database contains over 60, 000 neuron and glial geometries in 40 different brain regions for several different species, including rodent and human. This interface allows not only the modelling of any single neuron geometry, but also a network of neurons with other neurons and/or glial cells (Belov et al. 2016). To model a neuron from the database, users should download the standardised ASCII data file (in the SWC open source format for storing neuron morphologies) of the specific neuron or glial cell they wish to simulate from the database. The standard SWC format is a text file starting with a free-text header section (denoted by the symbol \#) and contains a matrix of 7 columns below the header (Cannon et al. 1998). The standard format defines four neuron components with an integer value (1 - soma, 2 - axon, 3 - basal dendrite and 4 - apical dendrite) while values of $5+$ are used for custom parameters. Note some formats extend on the standard format $(\mathrm{SWC}++)$ and include fork and end points as values 5 and 6 , respectively. The fields 
Table 1. The standard SWC format for neuron morphologies as used by the NeuroMorpho database.

\begin{tabular}{|c|c|c|}
\hline & Data Type & Data Value \\
\hline 1 & Integer & $\begin{array}{l}\text { Label that identifies the current point/structure. } \\
\text { Parent component (first point in each file) is always set to }-1 \text {. } \\
\text { Parent components are always less than child components. } \\
\text { Usually all trees originate from the soma. }\end{array}$ \\
\hline 2 & Integer & $\begin{array}{l}\text { The type of neuronal segment. Standard SWC format: } \\
0 \text { - undefined } \\
1 \text { - soma } \\
2 \text { - axon } \\
3 \text { - basal dendrite } \\
4 \text { - apical dendrite } \\
5+\text { - custom (user-defined preference) }\end{array}$ \\
\hline 3 & Double & $\mathrm{X}$ coordinate in micrometers \\
\hline 4 & Double & $\mathrm{Y}$ coordinate in micrometers \\
\hline 5 & Double & $\mathrm{Z}$ coordinate in micrometers \\
\hline 6 & Double & Radius of component in micrometers \\
\hline 7 & Double & $\begin{array}{l}\text { Parent sample. This value denotes connectivity with parent samples } \\
\text { appearing before child samples. }\end{array}$ \\
\hline
\end{tabular}

in the NeuroMorpho standard SWC files are further summarised in Table 1.

Generally, all neuron structures include a soma and all trees originate from the soma. In the rare case that a SWC file does not include a soma, the origination point of the tree will always be connected to the point that is defined as the parent. Soma in the SWC file format can consist of a single point or multiple points. When soma are encoded as a single line in the datafile, TOPAS-nBio will assign a sphere to the geometry (Figure 4a), if the soma is encoded as multiple lines, the soma will be made up of multiple spheres unionised into a single structure (for example see Figure 4b,c). The dendrites and axon components are made up of multiple cylinders of the specified radius in the SWC file, with the center of the cylinder specified by the spatial components (x,y,z) in the SWC file. To avoid geometric overlaps, which can cause errors in the Geant4 tracking, these cylinders are "cut" to fit next to each other without overlapping.

3.0.5. Specialised cells - osteoblasts, osteoclasts and osteocytes Bone specific complications such as bone loss (osteopenia), growth arrest, fracture and malignancy are often observed after irradiation (Hopewell 2003, Vassilopoulou-Sellin et al. 1999). Bone loss caused by weightlessness in zero-gravity being exacerbated from radiation exposure is also a major concern for astronauts on long-haul flights e.g., manned missions to Mars (Willey et al. 2011). Bone loss following radiation treatment was originally believed to be primarily caused from changes in the bone vasculature (Rohrer et al. 1979) but more recently, radiation induced damage to osteoblasts and osteocytes is thought to be a significant contributor (Noble \& Reeve 2000, Hopewell 2003). 




Figure 5. A model of an osteon in TOPAS-nBio. An osteon is cylindrical in shape consisting of concentric layers of compact bone tissue (blue). Three cell structures can be found in an osteon; osteoblasts which synthesise the bone matrix, osteocytes which are inactive osteoblasts trapped within the bone matrix and osteoclasts which are multi-nucleated cells that break down the bone matrix through phagocytosis.

Osteoblasts are the cells responsible for generating new bone matrix while osteoclasts are multi-nucleated cells that break down the bone matrix. Osteoblasts can also be converted into osteocytes, which are cells found inside the bone matrix, thought to play important roles in modulating signals that allow bone to adapt by either growing or breaking down (Noble \& Reeve 2000). The number of osteoblasts has been observed to be reduced after irradiation (Cao et al. 2011) while the number of osteoclasts is increased, coinciding with the observation of a reduction of bone matrix formation. The effect of radiation on osteocyte function and numbers is still not clear but the cells appear to be relatively radioresistant (Sugimoto et al. 1991). The underlying mechanisms leading to the increased activity of osteoclasts after irradiation is still not known (Macias et al. 2016). Track structure Monte Carlo simulations could aid in advancing our understanding of how radiation processes interact with these cells in bone tissue.

TOPAS-nBio includes geometry extensions for osteoblasts, osteocytes and osteoclasts namely TsOsteoblast, TsOesteocyte and TsOsteoclast. Figure 5 shows an example of a TOPAS-nBio simulation geometry of a bone matrix containing osteocytes randomly distributed in the matrix with both cuboidal osteoblasts and multi-nucleated osteoclasts lining the matrix. 


\subsection{Organelles}

Due to the stochastic nature of radiation energy deposition events, Monte Carlo simulations are a powerful tool to investigate the effects of radiation in sub-micron sized volumes such as organelles or molecules such as DNA.

Undeniably the nucleus and the genome it contains is the primary radiation target in the cell and of vital importance in most radiobiology studies. Accurate models of the spatial and temporal distribution of radiation induced damage, in the form of DSBs or lesions, on the full DNA structure and its successive evolution is essential for formulating a full understanding of the biological effects of radiation. Recent experimental evidence is, however, challenging this DNA-centric model and suggesting the full effects of radiation may not be initiated from DNA damage exclusively. Experiments in which the cytoplasm is irradiated, while the nucleus is spared, suggest that radiation induced damage in extra-nuclear cell structures may also be of importance for developing a comprehensive model of the cellular response to radiation (Deshpande et al. 1996, Wu et al. 2017). For this reason, we include full nuclear DNA models as well as other cellular organelles and structures in the TOPAS-nBio toolkit.

3.1.1. Nucleus The nucleus is the principal focus of most radiobiology studies. Users have the option of including a very simple nucleus in their cell models, usually modelled as a sphere (or ellipsoid). Users should specify the size of the nucleus as well as the position within the cell (see Figure 2). This simple model is useful for investigating the total dose received by the nucleus or can be used in combination with the TOPAS-nBio DBScan scorer to estimate the amount of DNA damage, without including a full DNA model.

Track structure simulations can be used to model damage to the DNA molecule in the form of single or double strand breaks and when combined with other biological repair models (McMahon et al. 2016, McMahon et al. 2017a, Ballarini et al. 2014) can predict the formation of lethal lesions and overall survival.

The human nucleus has a hierarchical structure starting from chromosome territories, chromatin domains, chromatin loops, chromatin fibres, nucleosomes down to the DNA double helix structure. These structures may all be of importance to the radiation-induced cellular response. For this reason, TOPAS-nBio gives users the ability to model the nucleus on a variety of levels. Two options for a full human genome model are offered, both including chromosome territories all the way down to the full DNA double helix structure. The first model is an adaption of the Geant4-DNA user application of a full nuclear model, while the second is based on a model of chromosome loops folded using a fractal.

3.1.2. Geant4-DNA full nuclear model The Geant4-DNA model represents the whole genome $\left(\sim 6 \times 10^{9} \mathrm{bps}\right)$ within an ellipsoid nucleus for a cell in the G0/G1 phase and is further described in Dos Santos et al. (2014). The DNA structure is divided into 



Figure 6. The Geant4-DNA nuclear DNA geometry in TOPAS-nBio: (a) shows the nucleus with chromosome territories represented by boxes, each filled with chromatin loops (pink "flower" substructures), (b) shows the arrangement of the chromatin fibres in a "flower" representing chromatin loops with each "petal" made up of 4 chromatin fibres and (c) shows the chromatin fibre, consisting of 90 nucleosomes. Each nucleosome consists of a histone (blue) wrapped by 2 turns of the DNA double helix (yellow and black strands).

5 organisation levels: chromosome territories, chromatin fibre loops, chromatin fibres, nucleosomes and the DNA double helix. The ellipsoid nucleus has half-axes of $13 \mu \mathrm{m}$, $10 \mu \mathrm{m}$ and $3 \mu \mathrm{m}$, representing a fibroblast.

The DNA double helix strands are composed of two separate strands built from the union of spheres. The sugar-phosphate backbone of the DNA has a total diameter of $2.16 \mathrm{~nm}$ and the DNA base, within the backbone structure, has a diameter of $0.34 \mathrm{~nm}$. The double helix is used to form a nucleosome which consists of a core histone protein (cylinder with diameter $6.5 \mathrm{~nm}$ and length of $5.7 \mathrm{~nm}$ ) wrapped by two turns of the DNA double helix (a total of $200 \mathrm{bps}$ ). The chromatin fibre is represented by a cylinder of diameter $30.8 \mathrm{~nm}$ and length $161 \mathrm{~nm}$. Each fibre contains 90 nucleosomes which are placed on a helix (Figure 6c). To represent the chromatin loops, 7 chromatin fibres are arranged in a "flower" shape. The flower has 7 "petals", with each composed of 4 fibres arranged in a diamond (Figure 6b). The flower substructures fill 23 chromosome territories, each represented by a box of varying size (Figure 6a). 



Figure 7. Illustration of a 3D Hilbert space filling curve for 4 iterations.

3.1.3. Fractal DNA Model An alternative full nuclear DNA model is provided in TOPAS-nBio based on the folding of the chromatin fibres in a fractal. It has been shown that at the mega-basepair scale, the chromatin folding is consistent with a so called knot-free fractal globule, which enables dense packing while preserving the ability to easily fold and unfold the genome (Lieberman-Aiden et al. 2009). The fractal globule is additionally consistent with other genome organisation features, including chromosome territories and sub-nuclear positioning. Fractal folding has also been used to model the genome of bacteria (Lampe et al. 2018).

In order to create a fractal globule with the same properties as that found in real DNA, a continuous 3D Hilbert space filling curve was used. A recursive function was used to generate the 3D Hilbert curve to fill a cube volume with a basic building block of an open cube formed with seven base components (or cylinders) as shown in Figure 7. The fractal pattern is made by recursively converting each line to a smaller version of the original starting pattern. A single iteration of the Hilbert curve forms 7 fibres, two iterations form 64 fibres, three iterations form 512 fibres, etc. Users have the option of specifying the number of iterations in a nucleus of any chosen size (Figure 8a), or to use the predefined full genome case (Figure 8b). Figure 8a, shows an example of a nucleus made with two iterations of the Hilbert curve forming 64 chromosome fibres, where each coloured region represents a chromosome territory. Each fibre further contains 90 nucleosomes, with each nucleosome containing a central histone protein wrapped with two turns of the DNA double helix. The histone protein is a cylinder with diameter 6.5 $\mathrm{nm}$ and length of $5.7 \mathrm{~nm}$. Two strands of DNA are formed with the union of spheres as described for the Geant4-DNA full nuclear model (Section 3.1.2). The sugar-phosphate 

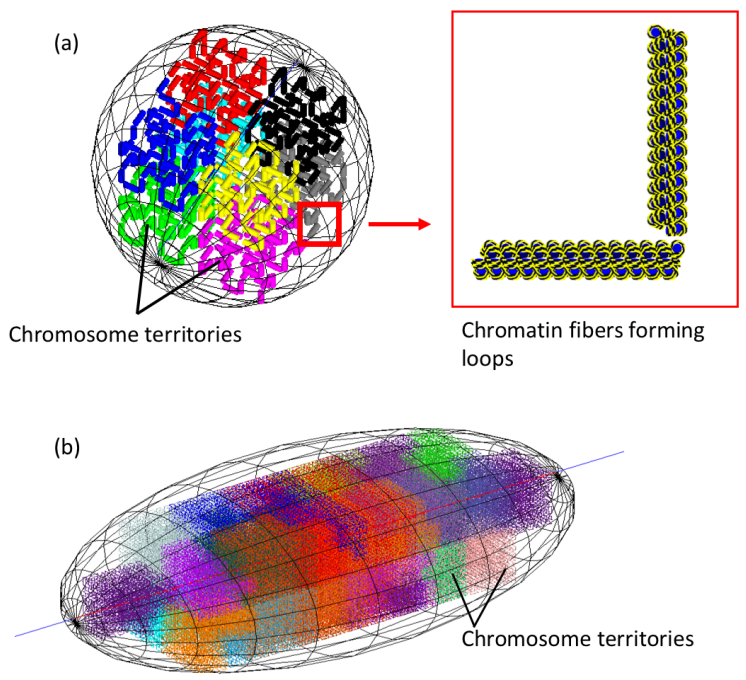

Figure 8. An example of the TOPAS-nBio fractal DNA model. (a) A spherical nucleus with 64 chromosome fibres arranged in an open cube, which is repeated using a 3D Hilbert space filling curve. The inserted panel shows the chromatin fibres, each containing 200 bps in a double helix (yellow and black), wrapped around histones (blue). (b) A TOPAS-nBio model of the cell nucleus with a full human genome with 23 chromosome territories. Chromatin fibres are folded using a 3D Hilbert space filling curve. The colours represent different chromosome territories.

backbone of the DNA has a total diameter of $2.16 \mathrm{~nm}$ and the DNA base has a diameter of $0.34 \mathrm{~nm}$. The fractal DNA model does allow users to specify other geometries for the chromatin fibre, some of these alternative models are further discussed in Section 3.2.

The full genome case consists of $\sim 342204$ chromatin fibres for the whole cell $\left(6 \times 10^{9} \mathrm{bps}\right)$. The fibres comprise 23 chromosome territories within an ellipsoid nucleus. The number of chromatin fibres (and basepairs) assigned to the 23 chromosomes are summarised in Table 2. The full genome case consists of seven separate Hilbert curves (to make up the exact number of fibres within the full genome) and arranged to fill the ellipsoid.

3.1.4. Mitochondria Mitochondria are the only sites of extra-nuclear DNA in eukaryotic cells and are essential for vital cellular function including energy production, apoptosis regulation, reactive oxygen species production and signalling. Experimental studies have shown that mitochondria are a potential radiation target in the cell (Kam \& Banati 2013, Kam et al. 2013) and various lesions (strand breaks, base mismatches and deletions) in the mitochondrial DNA can be induced by ionising radiation (Singh 
Table 2. An estimation of the number of DNA basepairs (bps) and chromatin fibres in each chromosome, based on data from the Genome Reference Consortium (GRC) https://www.ncbi.nlm.nih.gov/grc.

\begin{tabular}{cccc}
\hline Chromosome Territory & Genes & Total bps & Number of chromatin fibres \\
\hline 1 & 2000 & $247,199,719$ & 13733 \\
2 & 1300 & $242,751,149$ & 13286 \\
3 & 1000 & $199,446,827$ & 11080 \\
4 & 1000 & $191,263,063$ & 10626 \\
5 & 900 & $180,837,866$ & 10047 \\
6 & 1000 & $170,896,993$ & 9494 \\
7 & 900 & $158,821,424$ & 8823 \\
8 & 700 & $146,274,826$ & 8126 \\
9 & 800 & $140,442,298$ & 7802 \\
10 & 700 & $135,374,737$ & 7521 \\
11 & 1300 & $134,452,384$ & 7470 \\
12 & 1100 & $132,289,534$ & 7349 \\
13 & 300 & $114,127,980$ & 6340 \\
14 & 800 & $106,360,585$ & 5909 \\
15 & 600 & $100,338,915$ & 5574 \\
16 & 800 & $88,822,254$ & 4935 \\
17 & 1200 & $78,654,742$ & 4370 \\
18 & 200 & $76,117,153$ & 4229 \\
19 & 1500 & $63,806,651$ & 3545 \\
20 & 500 & $62,435,965$ & 3469 \\
21 & 200 & $46,944,323$ & 2608 \\
22 & 500 & $49,528,953$ & 2752 \\
Y & 50 & $57,741,652$ & 3208 \\
$\mathrm{X}$ & 800 & $154,913,754$ & 8606 \\
\hline & & &
\end{tabular}

et al. 1985, Rogounovitch et al. 2002). Irradiated mitochondria have been observed to have altered function that induces apoptosis (Taneja et al. 2001). Mitochondria may also play an important role in radiation-induced signalling (Tartier et al. 2007). Furthermore, microbeam studies that target the cytoplasm and hence likely include mitochondria, show increased damage in the nuclear DNA, suggesting mitochondria play an important role in mediating cytoplasmic radiation induced damage (Zhang et al. 2014).

Mitochondrial DNA (mt-DNA) in humans contains $\sim 16569$ bps encoding 37 genes and unlike nuclear DNA, which is linear, mt-DNA is circular. The mt-DNA encodes genes related to the oxidative phosphorylation system, which allows the mitochondria to produce energy for the cell. However, mitochondria depend on nuclear gene products as well. Each mitochondrion contains multiple copies of the mitochondrial genome, and each cell can contain many mitochondria, depending on the type of cell. Unlike nuclear DNA, mt-DNA is not enveloped or packaged into higher structures. In TOPAS-nBio the mt-DNA may be modelled using a circular or non-circular plasmid geometry. These geometries are further described in Sections 3.2.4 and 3.2.5.

It has been hypothesised that the observed dose enhancement effects leading to 
(a)

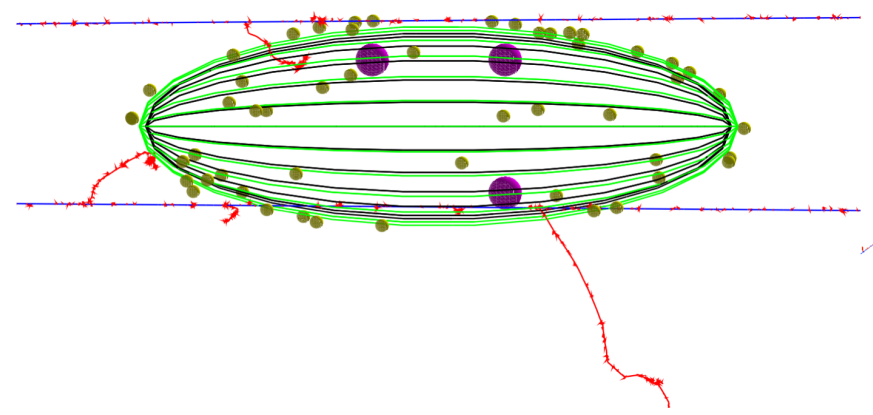

(b)



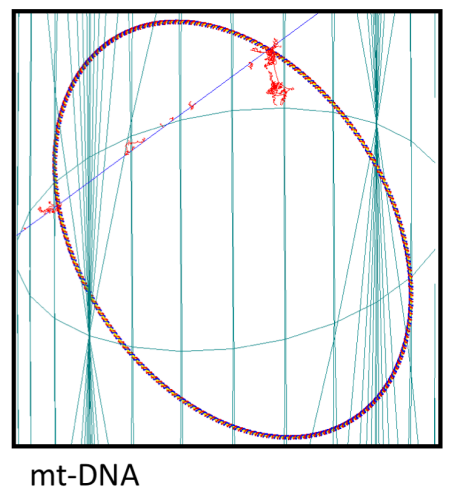

Figure 9. TOPAS-nBio simulations of mitochondria. (a) A mitochondrion with the outer membrane coated in gold nanoparticles to investigate dose enhancement effects on the organelle. Shown in blue is a proton track, with ionisation events shown in yellow, the purple spheres are volumes representing the mt-DNA. (b) A simulation of a mitochondrion including a model of the circular mt-DNA consisting of a double helix. A proton track is shown in blue, while secondary electron tracks are shown in red.

enhanced cell death from gold nanoparticles (GNPs), even when the GNPs do not enter the nucleus, may be attributed to the targeting of mitochondria instead of the nuclear DNA (McNamara et al. 2016, McMahon et al. 2017b, Kirkby \& Ghasroddashti 2015). Figure 9 (a) shows an example of a TOPAS-nBio simulation of a mitochondrion with GNPs attached to the outer membrane, in order to investigate the dose enhancement effect. Figure 9 (b) shows a mitochondrion with circular mt-DNA.

3.1.5. Dendrite spines and neuron organelles Irradiation of the CNS may result in serious detrimental side effects including cognitive dysfunction. Although radiationinduced brain injury is well chronicled, the main underlying mechanisms responsible for severe neurocognitive sequelae have not been determined.

Spines, small protrusions on the dendrite membrane, aid in storing and transmitting neurotransmitters and form a vital role in forming healthy synapses in the brain. Studies have shown morphological changes occur in spines after irradiation, with a significant reduction in both the number and density of spines (Parihar et al. 2015b). Other studies have additionally reported a loss of the protein drebrin after irradiation, an indication of synaptopathy (Puspitasari et al. 2016). Since compromised dendritic and spine morphologies are observed in many other significant neurodegenerative disorders (Kaufmann \& Moser 2000), it has been suggested to be a major contributing factor in radiation-induced cognitive dysfunction. Immature spine morphologies (thin, stubby) seem to be more radiosensitive than mature (mushroom) spines (Parihar et al. 2015b), indicating that geometry may be an important factor. Monte Carlo track structure 


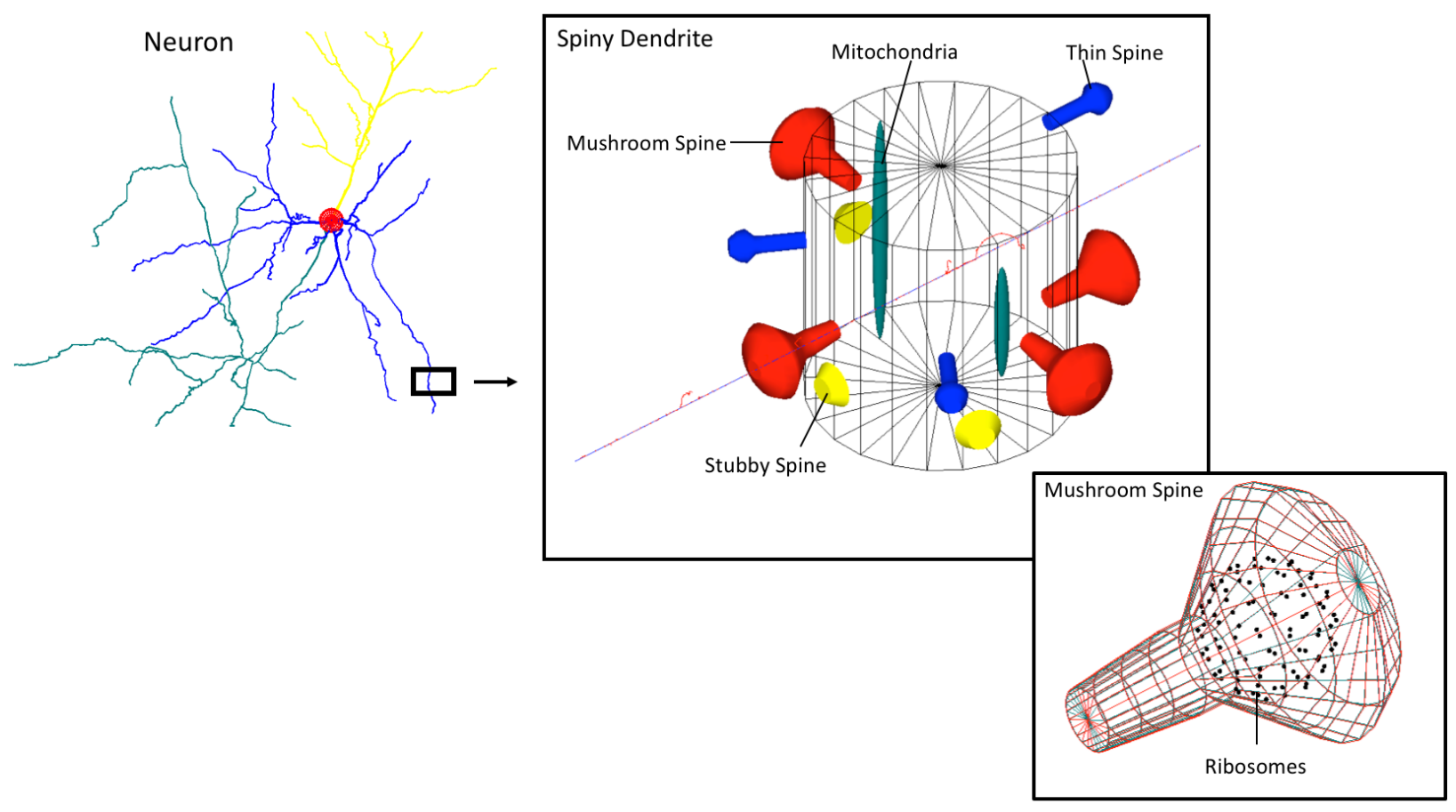

Figure 10. TOPAS-nBio models of a spiny dendrite. The dendrite contains three different spine morphologies: mushroom (red), thin (blue) and stubby (yellow) spines. In addition, the dendrite also includes mitochondria components (green) and ribosomes are shown in a mushroom spine. The central panel shows a proton track in green transversing the dendrite, secondary electron tracks are shown in red.

simulations in realistic neuron geometries, and specifically potential radiation targets such as the spines in dendrites, can predict the spatial distribution of energy depositions within these components (Alp et al. 2015). These microscopic dose depositions and diffusion of chemical reactive species, when correlated with experimental data, may aid in our understanding of the effects of radiation in the brain. TOPAS-nBio allows users to model three different spine morphologies; these include stubby, mushroom and thin spines. Additionally, TOPAS-nBio allows the simulation of other potential radiation targets in the dendrite, including molecules (e.g., ribosomes) and other organelles essential for normal synaptic function. Figure 10 shows an example of a dendrite section in TOPAS-nBio. The dendrite contains three different spine morphologies, namely mushroom, thin and stubby spines. Mitochondria are also included in the dendrite as well as ribosomes in a mushroom spine. 

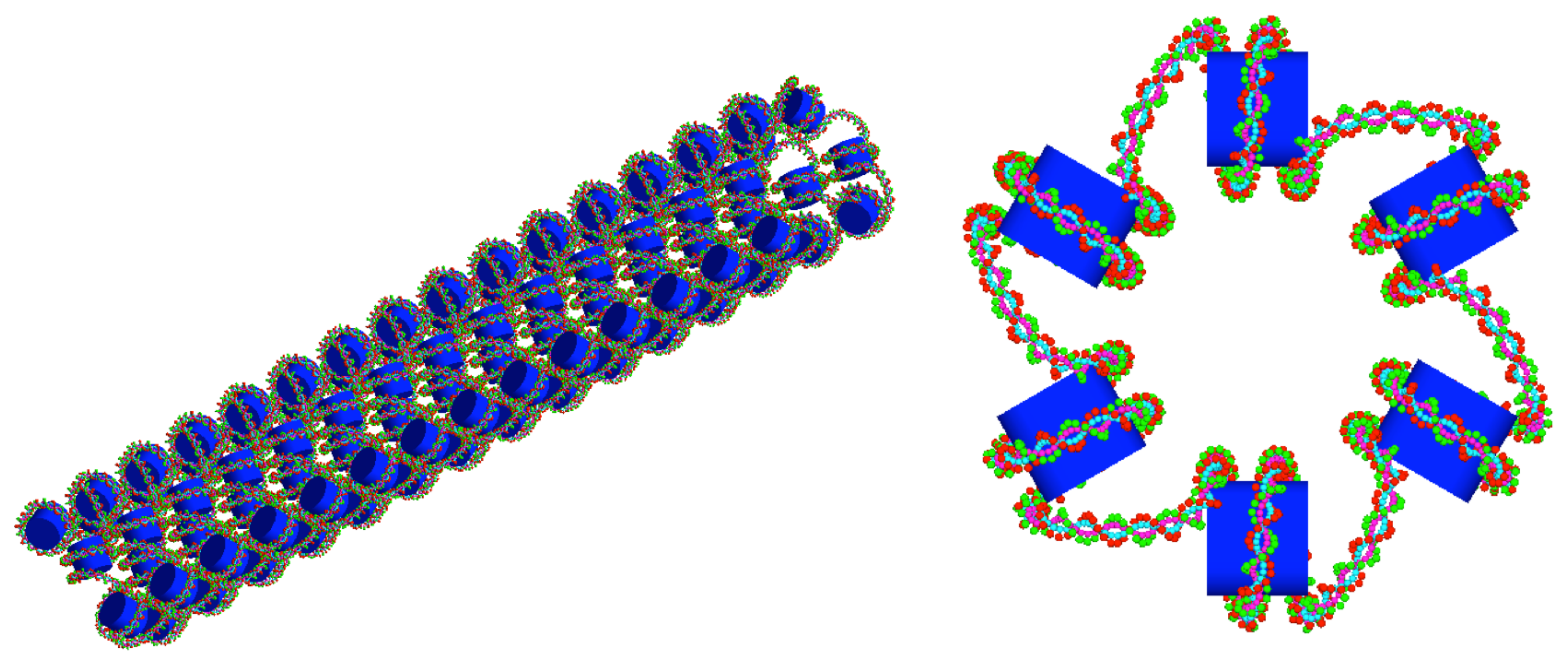

Figure 11. The solenoid chromatin fibre model based on Henthorn et al. (2017). Reprinted with permission from Radiation Research, 188, Henthorn NT, Warmenhoven JW, Sotiropoulos M, Mackay RI, Merchant MJ, Nanodosimetric Simulation of Direct Ion-Induced DNA Damage Using Different Chromatin Geometry Models, 690-703, Copyright 2017.

\subsection{DNA Fibres and Molecules}

In some cases, users may wish to simulate a single chromatin fibre or a strand of DNA or a DNA plasmid. TOPAS-nBio provides several geometries for each case. In addition, proteins and lipids are biologically significant molecules that users may wish to include in their simulation studies.

3.2.1. Solenoid Chromatin Fibre Model A chromatin fibre model, based on a solenoid geometry (Finch \& Klug 1976), is provided in TOPAS-nBio adapted from Henthorn et al. (2017). The model of the double helix has backbones and bases modelled as spheres wrapped around a cylindrical histone protein. The radius of the base sphere was $0.208 \mathrm{~nm}$, while the backbone sphere had a radius of $0.240 \mathrm{~nm}$. The histone radius was set to $3.3 \mathrm{~nm}$ with a length of $5.7 \mathrm{~nm}$. The double helix structure is wrapped around cylindrical histones in 1.65 left-handed turns to form the nucleosome. These are arranged in a solenoid chromatin conformation (see Figure 11). Each fibre contains 61 histones and $\sim 10.8 \mathrm{kbp}$ of DNA. 


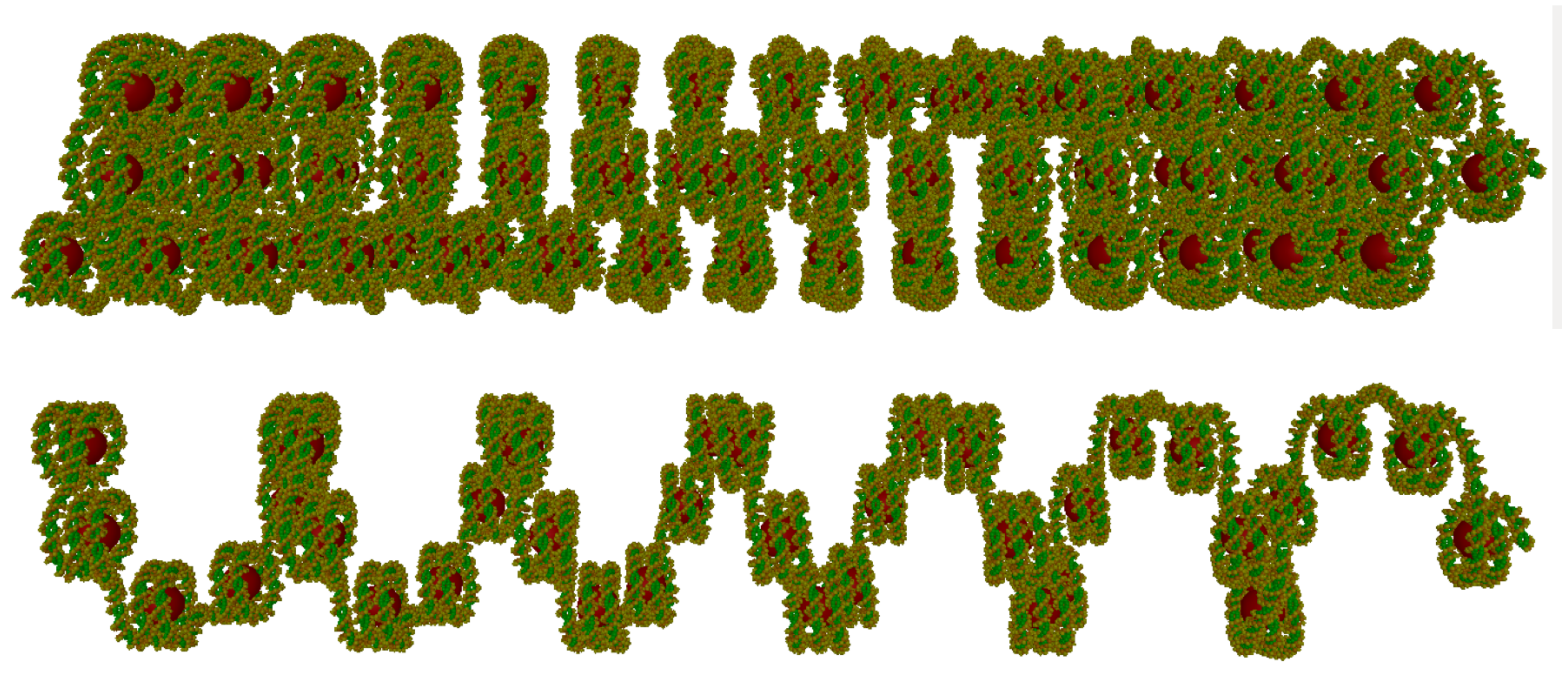

Figure 12. The DnaFabric chromatin model. Histones are shown in red, while the double helix structure is shown in green and gold. Reprinted from Computer Physics Communications, 204, S. Meylan,U. Vimont,S. Incerti,I. Clairand,C. Villagrasa, Geant4-DNA simulations using complex DNA geometries generated by the DnaFabric tool, 59-169, Copyright (2016), with permission from Elsevier.

3.2.2. DnaFabric Fibre Model The chromatin fibre model from the DnaFabric project (Meylan et al. 2016) may also be imported in TOPAS-nBio for modelling damage to DNA. The DNA fibre model, which is composed of molecular volumes representing the DNA sugar-phosphate backbone and bases, was created with DnaFabric with the file extension .dnafab. This file is then read into TOPAS-nBio and the data is converted into Geant4 volumes. After these volumes are placed in the world volume, an algorithm checks for overlaps between volumes and removes the overlapping regions.

The DNA fibre model has a diameter of $30 \mathrm{~nm}$ and is composed of nucleosomes and linkers placed in a helical pattern (see Figure 12). Further information on DnaFabric and the model can be found in Meylan et al. (2016).

3.2.3. Simple DNA models: cylindrical targets DNA has been modelled with Monte Carlo simulations for the last three decades. Originally, energy depositions were modelled within simple cylindrical targets representing DNA strands, nucleosomes or chromatin fibres in order to compare to the experimental microdosimetry data (Nikjoo et al. 1991, Nikjoo et al. 1989). To predict strand breaks within the DNA from track structure simulations, DNA double helix structures were composed of more than one cylinder, representing the sugar-phosphate backbone as well as the DNA 
basepair (Humm \& Charlton 1988). Atomic models of the DNA double helix structure were later developed (Pomplun 2009, Delage et al. 2015) and used to construct the higher structures of the nuclear genome (nucleosomes, chromatin fibres, etc.) (Tomita et al. 1994, Dos Santos et al. 2013, Meylan et al. 2016, Friedland et al. 2011).

We include some of these simple DNA structures as geometry extensions in TOPASnBio for users who may want to compare dose depositions with microdosimetric experimental data or with the vast collection of simulation data in the literature. In an earlier study, some of the models in TOPAS-nBio were validated against some of the available literature and experimental data (McNamara et al. 2017).

TOPAS-nBio includes three cylindrical targets as geometry extensions called TsChromatin, TsNucleosome and TsDNA. Each target is based on the rough dimensions for a DNA strand, a nucleosome and a chromatin (Nikjoo et al. 1991, Nikjoo et al. 1989) and is shown in Figure 13a. The diameter and length of the cylinder representing the DNA strand, nucleosome and chromatin are $2 \mathrm{~nm} \times 2 \mathrm{~nm}, 10 \mathrm{~nm} \times 5 \mathrm{~nm}$ and $25 \mathrm{~nm} \times 25 \mathrm{~nm}$, respectively.

To model strand breaks in simple DNA segments, several models are offered including the TsCharltonDNA, TsPlasmid and TsLinearDNA geometry extensions. The TsCharltonDNA model is based on a simple combination of cylinders (Charlton et al. 1989, Humm \& Charlton 1988). The inner cylinder has a diameter of $1 \mathrm{~nm}$ and length of $0.34 \mathrm{~nm}$, representing the basepair of the DNA strand. Two surrounding halfcylinders represent the sugar phosphate backbone of the DNA; these are each rotated by 36 degrees on adjacent base-pairs (see Figure 13b). A similar model called TsLinearDNA also models the DNA basepair as a cylinder of diameter $1 \mathrm{~nm}$ and length $0.34 \mathrm{~nm}$, but models the sugar phosphate backbone as two quarter cylinders opposite each other with an outer diameter of $2.37 \mathrm{~nm}$, rotated by 36 degrees on each subsequent basepair (see Figure 13d).

3.2.4. Circular Plasmids Plasmids are small, circular, double-stranded DNA molecules commonly used in radiation biology studies. Plasmids additionally naturally exist in bacterial cells, and they also occur in some eukaryotes. Additionally, mt-DNA may be modelled as a plasmid with the appropriate number of basepairs $\sim 16569 \mathrm{bps}$, since it does not contain any higher hierarchal folding. TOPAS-nBio offer the users a circular plasmid model called TsPlasmid, in which the number of basepairs to include in the model is specified within the parameter file. Each DNA segment consists of a central cylindrical basepair (diameter $1 \mathrm{~nm}$ and length $0.34 \mathrm{~nm}$ ) surrounded by two quarter cylinders (diameter $2.37 \mathrm{~nm}$ ) for the sugar phosphate backbone. Figure 13c shows an example of two circular plasmids consisting of 100 and 1000 basepairs.

3.2.5. Non-circular Plasmids The structure of the non-circular plasmid was simulated using the Vologodskii procedure (Klenin et al. 1991). Starting with a chain conformed of a regular polygon of 149 sides of $10 \mathrm{~nm}$ length, an iterative sampling process was performed by displacing the vertices from the previous conformation and evaluating the 
(a) Cylinders

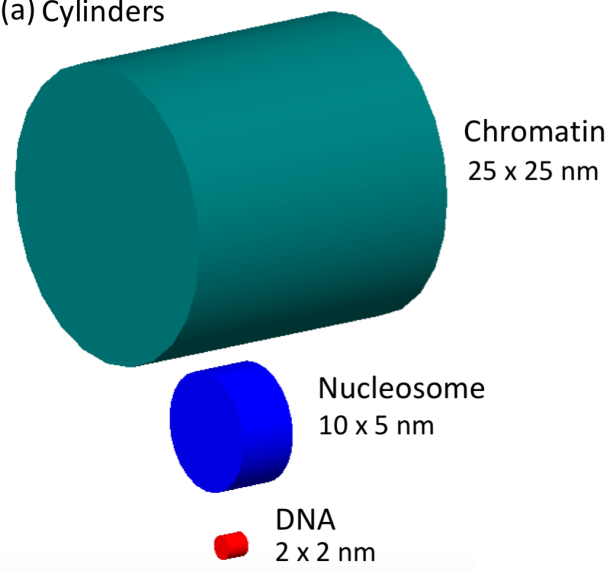

(b) Charlton DNA

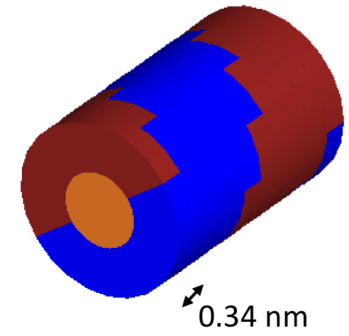

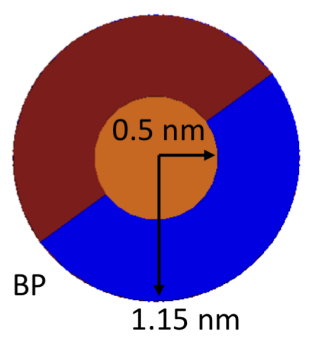

(c) Linear DNA

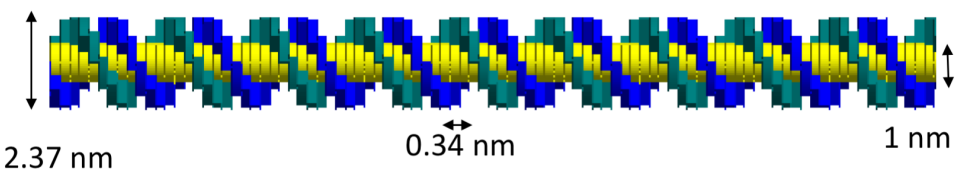

(d) Circular Plasmids

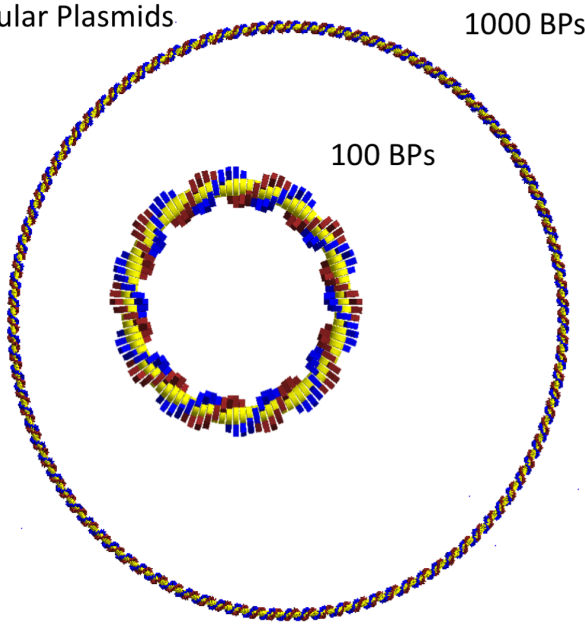

Figure 13. Simple DNA models in TOPAS-nBio. (a) Cylindrical targets for a chromatin, nucleosome and DNA strand with diameter $\mathrm{x}$ length given for each. (b) The Charlton DNA model consisting a sub-divided cylinder. Each basepair (orange) consists of a cylinder of length $0.34 \mathrm{~nm}$ and radius $0.5 \mathrm{~nm}$. Two half-cylinders with radius $1.15 \mathrm{~nm}$ are wrapped around the basepair and rotated by 36 degrees on subsequent basepairs. (c) Circular plasmid models consisting of 1000 and 100 basepairs. (d) A linear DNA strand, consisting of a central cylinder with diameter of $1 \mathrm{~nm}$. Two quarter cylinders (diameter $2.37 \mathrm{~nm}$ ) are wrapped around each basepair and rotated at 36 degrees from each other (same configuration as in c).

elastic energy of the chain defined as the sum of the bending and torsional energies (Klenin et al. 1991). The vertices displaced were randomly chosen and two types of displacement were considered: crankshaft rotation and sub-chain translation. When a trial conformation was created, it was tested for not overlapping nor knots. The conformation was accepted only if the elastic energy was smaller than from the previous conformation, otherwise it was accepted under an exponential probability distribution. The iteration is stopped when thermic equilibrium was achieved for a temperature of 298 K. DNA molecules are represented as semi-ellipsoids and are wrapped every 0.34 $\mathrm{nm}$ and 36 degrees around the plasmid structure. The parameters of the ellipsoids were configured to match the volume of molecules of a nucleotide pair reported in (Meylan et al. 2016) but without the use of Boolean solids and avoiding overlaps. Figure 14 




Figure 14. An example of two non-circular plasmid models in TOPAS-nBio. The DNA double helix structure is composed of spheres as shown in the middle panel.

shows an example of two non-circular plasmids in TOPAS-nBio.

Proteins and Nucleic acids The protein data bank (PDB) contains over 130,000 biological macromolecular 3D structures, including proteins and nucleic acids (https://www.rcsb.org). Many of these proteins are essential to normal cellular function, and precise modelling of the spatial distribution in a realistic molecular model can aid in extending our understanding of the effect of radiation.

A user application in Geant4 called PDB4DNA allows the simulation of energy deposition events within a volume generated from a PDB file (Delage et al. 2015). This powerful application allows users to simulate damage to any protein contained within the extensive data bank, in a realistic geometry. The PDB4DNA application has been adapted to TOPAS-nBio to create an interface for creating geometries from PDB files. PDB files are read into TOPAS and a geometry of the molecule is created, using spheres to represent each atom. An example of PDB proteins read into a TOPAS-nBio simulation is shown in Figure 15.

\subsection{Cell membranes}

All cells have an outer membrane, which regulates and protects the cell. Eukaryotic cell organelles are also encased by membranes, which control the exchange of substances in the cell. Generally, cell membranes are made up of a bilayer of glycerophospholipids, 




Figure 15. Molecules from the protein data bank read into TOPAS-nBio with a proton track (blue) and secondary electrons (red). The top panel shows two nucleic acids; a nucleosome

and an RNA strand. The bottom panel shows three molecules that could be of interest in radiobiology studies: a drebrin complex found in neuron dendrites, CTLA-4, which is important in immunotherapy and a cell membrane channel.

a molecule composed of glycerol, a phosphate group and two fatty acid chains. Each glyceropholipid molecule can be divided into two main regions, the hydrophilic head and the hydrophobic tail. This unique geometry ensures the lipids aggregate into bilayers with the hydrophilic phosphate heads on the outside. Embedded within the membranes are transmembrane proteins, including transport proteins to move specific molecules in and out of the cell and proteins with communication functions, for example binding hormones or immune mediators to their extracellular portions. Mitochondria have two surrounding membranes, with the inner membrane containing many proteins that play a role in energy generation in the electron transport chain.

Studies of irradiated membranes have shown that water radiolysis can lead to lipid peroxidation (Stark 1991). Lipid peroxidation results in a significant structural change in the membrane which affects both the permeability and the micro-viscosity of the lipids. Lipid peroxidation has been linked to many diseases and is possibly carcinogenic. The biological outcomes of the radiation-induced structural changes in membranes are however mostly unknown. Since many cellular processes are dependent on the proper functioning of channels in the membrane, ion protein channels could be radiation sensitive targets that are yet to be studied in detail. 




(b)

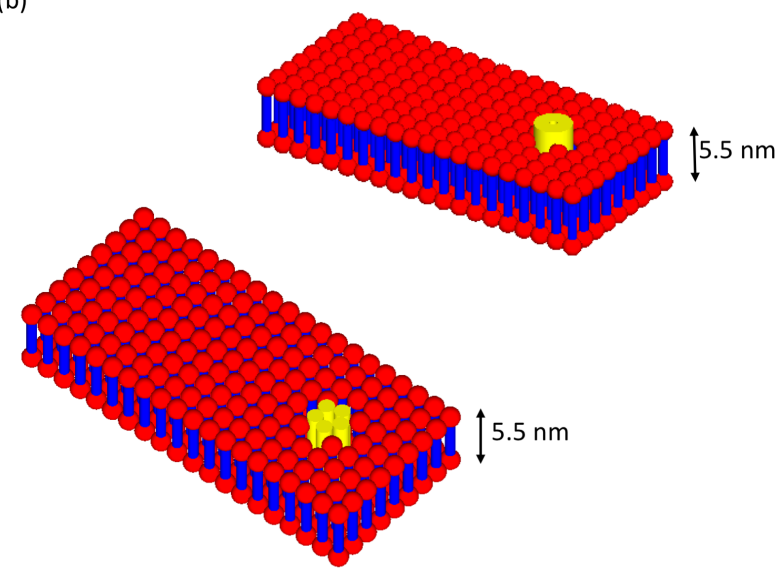

Figure 16. TOPAS-nBio models of the cell membrane. The lipid bilayer is made up of a hydrophilic head (red sphere) and the hydrophobic tail (blue cylinder). This lipid unit can be placed in different configurations: e.g. (a) in a ring formation or (b) in a layer. Users have the option of including transmembrane proteins; (b) shows two examples of the ion channel geometries (yellow), one consisting of 5 cylinders forming the subunits of the channel or a single cylindrical protein.

A lipid bilayer is created in TOPAS-nBio consisting of two hydrophilic heads, each a sphere of radius $2 \mathrm{~nm}$, and a tail region consisting of a cylinder of radius $1 \mathrm{~nm}$ and length of $2 \mathrm{~nm}$. All dimensions are changeable parameters. Figure 16 shows examples of membranes in TOPAS-nBio. The membrane can be formed as a ring (a), sphere or a layer (b) generated by the base elements i.e. the lipid unit. Users are also able to model the transmembrane proteins, which can represent ion channels or communication proteins (b). Ion channels are formed by three or more protein subunits (modelled as cylinders) arranged in a circle within the membrane. Communication proteins are modelled as a single cylinder. Channels may also be formed by a single cylinder with a hole drilled through its middle.

\subsection{Scorers}

For the majority of the geometries discussed above, a custom scorer has been designed for each. Generally, these are n-tuple scorers that may be further customised by the user. N-tuples contain the set of elements to be scored. This can include parameters such as position ( $\mathrm{x}, \mathrm{y}$ and $\mathrm{z}$ coordinates), energy deposition, kinetic energy of the interacting particle, interacting particle type, physics process type of the interaction and name 
(or ID) of the geometry component in which the interaction occurs. In some scorers, for example DNA models, quantities such as single or double strand breaks may be scored. Additionally, any of the scorers currently available in TOPAS can also be used in TOPAS-nBio to score quantities such as dose, for example. These are all controlled through the parameter file system. Users who wish to create their own custom scorers can do this by writing their own TOPAS scoring extension class.

Users of TOPAS-nBio may choose to make use of the TOPAS phasespace scorers. This allows users to score a set of particles crossing a specified surface which can then be re-read into TOPAS as a source. This is useful for multi-scale simulations. For example, in the case of an irradiated cell culture, users can simulate the full beamline of their experiment (e.g., proton beamline or linac) using condensed history simulations and generate a phasespace at a specified surface before the placement of their cell culture. This phase space can be "shrunken" using the tools of TOPAS to generate a new source for directly irradiating a single cell in a TOPAS-nBio simulation. The use of phasespace scorers in a complex experimental setup can save time and improve statistics in the scored Monte Carlo calculated quantities.

\section{Conclusions}

The TOPAS-nBio extension provides users with a powerful and easy-to-use tool for the development of advanced radiobiology Monte Carlo simulations. The extension allows the spatial distribution of energy depositions, as well as the diffusion of free radical species, from advanced track structure simulations to be modelled within realistic biological volumes. Here we present the geometries that have been developed to be included in the open-source TOPAS-nBio radiobiology toolkit.

\section{Acknowledgements}

This work was supported by the National Institutes of Health (NIH)/National Cancer Institute (NCI) grant R01 CA187003. The authors wish to thank Dudley Goodhead for helpful discussions regarding relevant biological targets.

\section{References}

Agostinelli S et al. 2003 Geant4 - a simulation toolkit Nucl. Instrum. Methods A 506, 250-303.

Alp M, Parihar V K, Limoli C L \& Cucinotta F A 2015 Irradiation of neurons with high-energy charged particles: an in silico modeling approach PLoS Comput Biol 11(8), e1004428.

Ballarini F, Altieri S, Bortolussi S, Carante M, Giroletti E \& Protti N 2014 The BIANCA model/code of radiation-induced cell death: application to human cells exposed to different radiation types Radiat. Environ. Bioph. 53(3), 525-533.

Batmunkh M, Belov O V, Bayarchimeg L, Lhagva O \& Sweilam N H 2015 Estimation of the spatial energy deposition in CA1 pyramidal neurons under exposure to $12 \mathrm{C}$ and $56 \mathrm{Fe}$ ion beams $J$. Radiat. Res. Appl. Sc. 8(4), 498-507. 
Belka C, Budach W, Kortmann R D \& Bamberg M 2001 Radiation induced CNS toxicity - molecular and cellular mechanisms Brit. J. Cancer 85(9), 1233-1239.

Belov O V, Batmunkh M, Incerti S \& Lkhagva O 2016 Radiation damage to neuronal cells: Simulating the energy deposition and water radiolysis in a small neural network Phys. Medica 32(12), 15101520.

Bernal M A, Bordage M, Brown J, Davidkova M, Delage E, El Bitar Z et al. 2015 Track structure modeling in liquid water: a review of the Geant4-DNA very low energy extension of the Geant4 Monte Carlo simulation toolkit Phys. Medica 31(8), 861-874.

Bug M U, Yong Baek W, Rabus H, Villagrasa C, Meylan S \& Rosenfeld A B 2017 An electron-impact cross section data set $(10 \mathrm{eV}-1 \mathrm{keV})$ of DNA constituents based on consistent experimental data: A requisite for Monte Carlo simulations Radiat. Phys. Chem. 130, 459-479.

Byrne H L, Domanova W, McNamara A L, Incerti S \& Kuncic Z 2015 The cytoplasm as a radiation target: an in silico study of microbeam cell irradiation Phys. Med. Biol. 60(6), 2325-2337.

Cannon R C, Turner D A, Pyapali G K \& Wheal H V 1998 An on-line archive of reconstructed hippocampal neurons J. Neurosci. Methods 84(1-2), 49-54.

Cao X, Wu X, Frassica D, Yu B, Pang L, Xian L, Wan M, Lei W, Armour M, Tryggestad E, Wong J, Wen C Y, Lu W W \& Frassica F J 2011 Irradiation induces bone injury by damaging bone marrow microenvironment for stem cells P Natl Acad Sci USA 108(4), 1609-1614.

Carrier J F, Archambault L, Beaulieu L \& Roy R 2004 Validation of Geant4, an object-oriented Monte Carlo toolkit, for simulations in medical physics Med. Phys. 31(3), 484-492.

Charlton D E, Nikjoo H \& Humm J L 1989 Calculation of initial yields of single- and double-strand breaks in cell nuclei from electrons, protons and alpha particles Int. J. Radiat. Biol. 56(1), 1-19.

Delage E, Pham Q T, Karamitros M, Payno H, Štěpán V, Incerti S et al. 2015 PDB4DNA: Implementation of DNA geometry from the Protein Data Bank (PDB) description for Geant4DNA Monte-Carlo simulations Comput. Phys. Commun. 192, 282-288.

Deshpande A, Goodwin E H, Bailey S M, Marrone B L \& Lehnert B E 1996 Alpha-Particle-Induced Sister Chromatid Exchange in Normal Human Lung Fibroblasts: Evidence for an Extranuclear Target Radiat. Res. 145(3), 260.

Dos Santos M, Villagrasa C, Clairand I \& Incerti S 2013 Influence of the DNA density on the number of clustered damages created by protons of different energies Nucl Instrum Meth B 298, 47-54.

Dos Santos M, Villagrasa C, Clairand I \& Incerti S 2014 Influence of the chromatin density on the number of direct clustered damages calculated for proton and alpha irradiations using a Monte Carlo code Progress in Nuclear Science and Technology 4, 449-453.

Douglass M, Bezak E \& Penfold S 2012 Development of a randomized 3D cell model for Monte Carlo microdosimetry simulations Med. Phys. 39(6Part1), 3509-3519.

Finch J T \& Klug A 1976 Solenoidal model for superstructure in chromatin P Natl Acad Sci USA 73(6), 1897-1901.

Friedland W, Dingfelder M, Kundrát P \& Jacob P 2011 Track structures, DNA targets and radiation effects in the biophysical Monte Carlo simulation code PARTRAC Mutat. Res. 711(1-2), 28-40.

Gorlia T, Stupp R, Brandes A A, Rampling R R, Fumoleau P, Dittrich C, Campone M M, Twelves C C, Raymond E, Hegi M E, Lacombe D \& van den Bent M J 2012 New prognostic factors and calculators for outcome prediction in patients with recurrent glioblastoma: a pooled analysis of EORTC Brain Tumour Group phase I and II clinical trials Eur. J. Cancer 48(8), 1176-1184.

Greene-Schloesser D, Robbins M E, Peiffer A M, Shaw E G, Wheeler K T \& Chan M D 2012 Radiationinduced brain injury: a review Front. Oncol. 2, 73.

Grinde M T, Vik J, Camilio K A, Martinez-Zubiaurre I \& Hellevik T 2017 Ionizing radiation abrogates the pro-tumorigenic capacity of cancer-associated fibroblasts co-implanted in xenografts Sci. Rep. 7, 46714.

Henthorn N T, Warmenhoven J W, Sotiropoulos M, Mackay R I, Kirkby K J \& Merchant M J 2017 Nanodosimetric simulation of direct ion-induced DNA damage using different chromatin geometry models Radiat. Res. 188(6), 770-783. 
Hopewell J W 2003 Radiation-therapy effects on bone density Med. Pediatr. Oncol. 41(3), 208-211.

Humm J L \& Charlton D E 1988 in 'DNA damage by Auger emitters' Taylor and Francis London pp. 111-122.

Incerti S, Douglass M, Penfold S, Guatelli S \& Bezak E 2016 Review of Geant4-DNA applications for micro and nanoscale simulations Phys. Medica 32(10), 1187-1200.

Incerti S, Ivanchenko A, Karamitros M, Mantero A, Moretto P, Tran H N, Mascialino B, Champion C, Ivanchenko V N, Bernal M A, Francis Z, Villagrasa C, Baldacchino G, GUÈYE P, Capra R, Nieminen P \& Zacharatou C 2010 Comparison of Geant4 very low energy cross section models with experimental data in water Med. Phys. 37(9), 4692-4708.

Incerti S et al. 2018 Geant4?DNA example applications for track structure simulations in liquid water: a report from the Geant4?DNA Project Med. Phys. .

Jorgensen P, Edgington N P, Schneider B L, Rupes I, Tyers M \& Futcher B 2007 The size of the nucleus increases as yeast cells grow Mol. Biol. Cell 18(9), 3523-3532.

Kam W W Y \& Banati R B 2013 Effects of ionizing radiation on mitochondria. Free Radical Bio. Med. 65, 607-619.

Kam W W Y, McNamara A L, Lake V, Banos C, Davies J B, Kuncic Z \& Banati R B 2013 Predicted ionisation in mitochondria and observed acute changes in the mitochondrial transcriptome after gamma irradiation: a Monte Carlo simulation and quantitative PCR study Mitoch. 13(6), 736742 .

Kaufmann W E \& Moser H W 2000 Dendritic anomalies in disorders associated with mental retardation Cereb. Cortex 10(10), 981-991.

Kirkby C \& Ghasroddashti E 2015 Targeting mitochondria in cancer cells using gold nanoparticleenhanced radiotherapy: a Monte Carlo study Med. Phys. 42(2), 1119-1128.

Klenin K V, Vologodskii A V, Anshelevich V V, Dykhne A M \& Frank-Kamenetskii M D 1991 Computer simulation of DNA supercoiling J. Mol. Biol. 217(3), 413-419.

Lampe N, Karamitros M, Breton V, Brown J M C, Sakata D, Sarramia D \& Incerti S 2018 Mechanistic DNA damage simulations in Geant4-DNA part 2: electron and proton damage in a bacterial cell. Phys. Medica .

Laurent T, Murase D, Tsukioka S, Matsuura T, Nagamori S \& Oda H 2012 A novel human hepatoma cell line, FLC-4, exhibits highly enhanced liver differentiation functions through the threedimensional cell shape J. Cell Physiol. 227(7), 2898-2906.

Liao D, Luo Y, Markowitz D, Xiang R \& Reisfeld R A 2009 Cancer associated fibroblasts promote tumor growth and metastasis by modulating the tumor immune microenvironment in a $4 \mathrm{~T} 1$ murine breast cancer model PLoS One 4(11), e7965.

Lieberman-Aiden E, van Berkum N L, Williams L, Imakaev M, Ragoczy T, Telling A, Amit I, Lajoie B R, Sabo P J, Dorschner M O, Sandstrom R, Bernstein B, Bender M A, Groudine M, Gnirke A, Stamatoyannopoulos J, Mirny L A, Lander E S \& Dekker J 2009 Comprehensive mapping of long-range interactions reveals folding principles of the human genome Science $\mathbf{3 2 6}$ (5950), 289293.

Macias B R, Lima F, Swift J M, Shirazi-Fard Y, Greene E S, Allen M R, Fluckey J, Hogan H A, Braby L, Wang S \& Bloomfield S A 2016 Simulating the lunar environment: partial weightbearing and high-LET radiation-induce bone loss and increase sclerostin-positive osteocytes Radiat. Res. 186(3), 254-263.

Makale M T, McDonald C R, Hattangadi-Gluth J A \& Kesari S 2017 Mechanisms of radiotherapyassociated cognitive disability in patients with brain tumours Nat. Rev. Neurol. 13(1), 52-64.

McMahon S J, McNamara A L, Schuemann J, Paganetti H \& Prise K M 2017a A general mechanistic model enables predictions of the biological effectiveness of different qualities of radiation Sci. Rep. 7(1), 10790.

McMahon S J, McNamara A L, Schuemann J, Prise K M \& Paganetti H 2017b Mitochondria as a target for radiosensitisation by gold nanoparticles J. Phys. Conf. Ser. 777(1), 012008.

McMahon S J, Schuemann J, Paganetti H \& Prise K M 2016 Mechanistic modelling of DNA repair and 
cellular survival following radiation-induced DNA damage Sci. Rep. 6(1), 33290.

McNamara A, Geng C, Turner R, Méndez J R, Perl J, Held K, Faddegon B, Paganetti H \& Schuemann J 2017 Validation of the radiobiology toolkit TOPAS-nBio in simple DNA geometries Phys. Medica 33, 207-215.

McNamara A L, Kam W W Y, Scales N, McMahon S J, Bennett J W, Byrne H L, Schuemann J, Paganetti H, Banati R \& Kuncic Z 2016 Dose enhancement effects to the nucleus and mitochondria from gold nanoparticles in the cytosol Phys. Med. Biol. 61(16), 5993-6010.

Meyers C A 2000 Neurocognitive dysfunction in cancer patients Oncology 14(1), 75-9.

Meylan S, Vimont U, Incerti S, Clairand I \& Villagrasa C 2016 Geant4-DNA simulations using complex DNA geometries generated by the DnaFabric tool Comput. Phys. Commun. 204, 159-169.

Nikjoo H, Goodhead D T, Charlton D E \& Paretzke H G 1989 Energy deposition in small cylindrical targets by ultrasoft X-rays Phys. Med. Biol. 34(6), 691-705.

Nikjoo H, Goodhead D T, Charlton D E \& Paretzke H G 1991 Energy deposition in small cylindrical targets by monoenergetic electrons Int. J. Radiat. Biol. 60(5), 739-756.

Noble B S \& Reeve J 2000 Osteocyte function, osteocyte death and bone fracture resistance Mol. Cell Endocrinol. 159(1-2), 7-13.

Parihar V K, Allen B, Tran K K, Macaraeg T G, Chu E M, Kwok S F, Chmielewski N N et al. 2015a What happens to your brain on the way to Mars? Sci. Adv. 1(4), e1400256-e1400256.

Parihar V K, Pasha J, Tran K K, Craver B M, Acharya M M \& Limoli C L 2015b Persistent changes in neuronal structure and synaptic plasticity caused by proton irradiation Brain Struct. Funct. 220(2), 1161-1171.

Perl J, Shin J, Schümann J, Faddegon B \& Paganetti H 2012 TOPAS: An innovative proton Monte Carlo platform for research and clinical applications Med. Phys. 39(11), 6818 - 6837.

Plante I \& Cucinotta F A 2013 'Multiple CPU Computing: The Example of the Code RITRACKS' Springer Berlin Heidelberg Berlin, Heidelberg.

Pomplun E 2009 A New DNA Target Model for Track Structure Calculations and Its First Application to I-125 Auger Electrons Int. J. Radiat. Biol. 59(3), 625-642.

Puspitasari A, Koganezawa N, Ishizuka Y, Kojima N, Tanaka N, Nakano T \& Shirao T 2016 X irradiation induces acute cognitive decline via transient synaptic dysfunction Radiat. Res. 185(4), 423-430.

Ramos-Méndez J, Perl J, Schuemann J, McNamara A, Paganetti H \& Faddegon B 2018 Monte Carlo simulation of chemistry following radiolysis with TOPAS-nBio Phys. Med. Biol. in press, - .

Ramos-Méndez J, Schuemann J, Incerti S, Paganetti H, Schulte R \& Faddegon B 2017 Flagged uniform particle splitting for variance reduction in proton and carbon ion track-structure simulations Phys. Med. Biol. 62(15), 5908-5925.

Rogounovitch T I, Saenko V A, Shimizu-Yoshida Y, Abrosimov A Y, Lushnikov E F, Roumiantsev P O, Ohtsuru A, Namba H, Tsyb A F \& Yamashita S 2002 Large deletions in mitochondrial DNA in radiation-associated human thyroid tumors Cancer Res. 62(23), 7031-7041.

Rohrer M D, Kim Y \& Fayos J V 1979 The effect of cobalt-60 irradiation on monkey mandibles Oral Surg. Oral Med. Oral Pathol. Oral Radiol. 48(5), 424-440.

Rosenbluth M J, Lam W A \& Fletcher D A 2006 Force Microscopy of nonadherent cells: a comparison of leukemia cell deformability Biophys. J. 90(8), 2994-3003.

Saury J M G \& Emanuelson I 2011 Cognitive consequences of the treatment of medulloblastoma among children Pediatr. Neurol. 44(1), 21-30.

Singh G, Hauswirth W W, Ross W E \& Neims A H 1985 A method for assessing damage to mitochondrial DNA caused by radiation and epichlorohydrin Mol. Pharmacol. 27(1), 167-170.

Stark G 1991 The effect of ionizing radiation on lipid membranes. Biochim. Biophys. Acta 1071(2), 103122.

Sugimoto M, Takahashi S, Toguchida J, Kotoura Y, Shibamoto Y \& Yamamuro T 1991 Changes in bone after high-dose irradiation. Biomechanics and histomorphology J. Bone Joint Surg. [Br] 73(3), 492-497. 
Taneja N, Tjalkens R, Philbert M A \& Rehemtulla A 2001 Irradiation of mitochondria initiates apoptosis in a cell free system Oncogene 20(2), 167-177.

Tartier L, Gilchrist S, Burdak-Rothkamm S, Folkard M \& Prise K M 2007 Cytoplasmic irradiation induces mitochondrial-dependent 53BP1 protein relocalization in irradiated and bystander cells Cancer Res. 67(12), 5872-5879.

Tomita H, Kai M, Kusama T, Aoki Y \& Ito A 1994 Monte Carlo simulation of DNA strand breaks induced by monoenergetic electrons using higher-order structure models of DNA Int. J. Radiat. Biol. 66(6), 669-682.

Uehara S, Nikjoo H \& Goodhead D T 1993 Cross-sections for water vapour for the Monte Carlo electron track structure code from $10 \mathrm{eV}$ to the $\mathrm{MeV}$ region Phys. Med. Biol. 38(12), 1841-1858.

Vassilopoulou-Sellin R, Brosnan P, Delpassand A, Zietz H, Klein M J \& Jaffe N 1999 Osteopenia in young adult survivors of childhood cancer Med. Pediatr. Oncol. 32(4), 272-278.

Willey J S, Lloyd S A J, Nelson G A \& Bateman T A 2011 Ionizing radiation and bone loss: space exploration and clinical therapy applications Clin. Rev. Bone Miner. Metab. 9(1), 54-62.

Wu J, Zhang B, Wuu Y R, Davidson M M \& Hei T K 2017 Targeted cytoplasmic irradiation and autophagy Mutat. Res. 806, 88-97.

Zhang B, Davidson M M \& Hei T K 2014 Mitochondria regulate DNA damage and genomic instability induced by high LET radiation Adv. Space Res-Series 1, 80-88. 